\title{
TREPONEMA DENTICOLA AND PORPHYROMONAS GINGIVALIS AS BIOINDICATOR ORAL HYGIENE STATUS AND ORGANOLEPTIC SCORE IN MOUTH BREATHING CHILDREN
}

\author{
SYAHFINA FARAHMIDA ALJOGJA, ARIADNA ADISATTYA DJAIS, CITRA FRAGRANTIA THEODOREA*
}

\author{
Department of Oral Biology, Faculty of Dentistry, Universitas Indonesia, Jakarta 10430, Indonesia. Email: citraob@gmail.com
}

Received: 26 September 2019, Revised and Accepted: 24 December 2019

ABSTRACT

Objective: Mouth breathing is a bad habit that has several impacts on dentocraniofacial growth and development in children. It also related to another oral cavity condition, such as poor oral hygiene and halitosis. Halitosis is caused by an anaerobic bacteria product such as Treponema denticola and Porphyromonas gingivalis. These bacteria are Gram-negative anaerobic bacteria that play a significant role to halitosis occurrence. The objective of this study is to determine the prevalence of T. denticola and P. gingivalis as bioindicator in mouth breathing children.

Methods: A total number of 60 subjects had a mouth breathing test (19 subjects diagnosed as mouth breathers and 41 subjects as nose breathers). Then, the subjects were classified into halitosis and oral hygiene status category. Identification of T. denticola and P. gingivalis in supragingival plaque and buccal mucosa subjects was used a conventional polymerase chain reaction method.

Results: The correlation between Oral Hygiene Index-Simplified and organoleptic score in mouth breathers has positive correlation ( $\mathrm{r}=0.001)$, in the contrary, in nose breathers, it has negative correlation $(\mathrm{r}=-0.046)$. Meanwhile, the prevalence of T. denticola and P. gingivalis in mouth and nose breathers has no significant differences. Moreover, the significance value of prevalence T. denticola and $P$. gingivalis based on clinical parameters halitosis and oral hygiene status has no differences.

Conclusion: The prevalence of T. denticola and P. gingivalis cannot be used as bioindicator in mouth breathers.

Keywords: Halitosis, Mouth breathing, Oral hygiene status, Organoleptic, Porphyromonas gingivalis, Treponema denticola.

(c) 2020 The Authors. Published by Innovare Academic Sciences Pvt Ltd. This is an open access article under the CC BY license (http://creativecommons. org/licenses/by/4. 0/) DOI: http://dx.doi.org/10.22159/ijap.2020.v12s1.37421

\section{INTRODUCTION}

Nose is a substantial organ body in a way brings the inhaled air under an ideal condition for the respiration [1]. According to Moss's theory of matrix functional, respiration through the nose has a significant role in growth and development of an ideal craniofacial structure. However, in some conditions, respiration through the nose could be altered to mouth breathing as a result of pathological adaptation toward nasal obstruction or another mechanical factor [2].

Mouth breathing can lead to dentocraniofacial growth alteration and oral cavity problems, such as halitosis and poor oral hygiene. The oral mucosa of mouth breathers will encounter an evaporation and oral mucosa becomes dry. This is a predisposition factor to halitosis and poor oral hygiene [3].

Bad breath or halitosis is an unpleasant smell for the individual and their surroundings [4]. Halitosis does not only occur in adults but it can also occur in children [5]. Sources of halitosis can be classified from the intraoral and extraoral. According to the study, the prevalence of intraoral halitosis is $85 \%$, the rest of it is extraoral, such as digestive and respiratory systems [6]. Halitosis has a multifactorial etiology, but the main cause is due to poor oral hygiene and volatile sulfur compounds (VSCs) gas generated by anaerobic bacteria. These conditions can occur by increasing of plaque accumulation and bacterial growth which results in decreased of salivary flow rate. The protection from the salivary capacity, such as self-cleaning, antibacterial, and buffering activities is inhibited, thus deteroriating the status of oral hygiene [4].

According to Saini, the activity of proteolytic anaerobic bacteria mainly generates VSCs with $90 \%$ of its major components consist methyl mercaptan $\left(\mathrm{CH}_{3} \mathrm{SH}\right)$, hydrogen sulfide $\left(\mathrm{H}_{2} \mathrm{~S}\right)$, and dimethyl sulfide $\left[\left(\mathrm{CH}_{3}\right)_{2} \mathrm{~S}\right][4,7]$. These gas-producing anaerobic Gram-negative bacteria include Treponema denticola, Porphyromonas gingivalis, Fusobacterium nucleatum, and Tannerella forsythia [8]. The bacteria will degrade protein compounds derived from saliva, gingival crevicular fluid, plaque, tongue dorsal, and desquamated epithelial cells [7].

T. denticola and P. gingivalis were found frequently in deep periodontal pockets, but it can be found in the supragingival area and oral mucosa $[8,9]$. T. denticola and P. gingivalis have a role in plaque formation as late colonizers. Bacterial colonization will affect its phenotypes, which is bacterial becomes more resistant to antibacterial agent than plankton bacteria [10]. The presence of these bacteria in healthy periodontal tissue is a periodontal disease risk factor [9]. Plaque accumulation can be evaluated using the instrument, like Oral Hygiene Index-Simplified (OHI-S). OHI-S score becomes a guideline to determine oral hygiene status [11].

Based on the above explanation, in this study, identification of T. denticola and P. gingivalis was carried out in mouth breathing children. T. denticola and P. gingivalis were assumed as biologic indicator for mouth breathing children and as an early warning toward the risk of periodontal disease in children.

\section{METHODS}

\section{Sample collection}

This study was approved by Ethics Committee, Faculty of Dentistry, Universitas Indonesia, with letter numbers 58/Ethical Approval/ FKGUI/VII/2019. Sixty subjects were recruited from SD Tugu Ibu, Depok. Participants and their parents were received an explanation about the aim and procedures of this study and agreed to participate in this study by assigned a written paper as informed consent. Sixty subjects were performed mouth breathing test, 19 subjects diagnosed as mouth breathers, and 41 as nose breathers. Children with mental 
issue, lip and palate cleft, and consumed antibiotics or antihistamine for the past 3 months were excluded from this study. The participants were classified based on oral hygiene status and organoleptic score. Participants were performed an organoleptic test using a carton paper as privacy screen and straw. Participants were asked to exhale slowly near the straw. Two operators assessed the smell with score range of $0-5$. Assessment performed in three different distances, $10 \mathrm{~cm}, 30 \mathrm{~cm}$, and $100 \mathrm{~cm}$. Subjects have organoleptic score $<2$ considered who had no halitosis; meanwhile, subjects have organoleptic score $\geq 2$ considered who had halitosis. Furthermore, oral hygiene assessment was used according to OHI-S Greene and Vermillion criteria [11]. OHI-S score classified subjects into three categories, good oral hygiene (0-1.2), moderate (1.3-3.0), and poor (3.0-6.0). Buccal mucosa samples were collected using a sterile brush to swab buccal mucosa 3 times each subject. Supragingival plaque samples were collected by pooling using a periodontal probe. The samples placed in $1 \mathrm{ml}$ phosphate-buffered saline (PBS) in microcentrifuge tube.

\section{Bacterial growth conditions}

T. denticola (ATCC 35404) and P. gingivalis (ATCC 33277) cultivated in brain heart infusion broth under anaerobic condition $\left(\mathrm{N}_{2} 80 \% ; \mathrm{H}_{2} 10 \%\right.$; $\mathrm{CO}_{2} 10 \%$ ) at $37^{\circ} \mathrm{C}$ for 3 days.

\section{DNA extraction}

DNA was extracted using heat shock method. The samples were washed using PBS 2 times and once using nuclease-free water. Samples were incubated in $100^{\circ} \mathrm{C}$ using a floating bath in a water bath for $20 \mathrm{~min}$. Then, the samples immediately placed in cooler box contain dry ice for $10 \mathrm{~min}$. DNA isolation was carried out by centrifugation at $10.000 \mathrm{rpm}$ for 5 min then taken out the supernatant.

\section{Identification T. denticola and $P$. gingivalis}

A pair of specific primer was used for each bacterium. For identification, T. denticola was used: Td1394-F (5'-AGAGCAAGCTCTCCCTTACCGT-3') and Td-1498-R (5'-TAAGGGCGGCTTGAAATAATGA-3'); meanwhile, P. gingivalis was used: Pg1198-F (5'-TACCCATCGTCGCCTTGGT-3') and Pg1323-R (5'-CGGACTAAAACCGCATACACTTG-3') [12].

\section{Polymerase chain reaction (PCR) protocol}

Reaction mixture consisted of $12.5 \mu$ l MyTaq, Red Mix ${ }^{\mathrm{TM}}, 0.5 \mu \mathrm{l}$ forward and reverse primer $5 \mu \mathrm{M}, 3 \mu \mathrm{l}$ DNA sample, and $3.5 \mu$ l double-distilled water. The total volume for each PCR mixture was $25 \mu$ l. PCR reactions were performed under these following conditions: Initial denaturation at $95^{\circ} \mathrm{C}$ for $2 \mathrm{~min}$, followed by 36 cycles of denaturation at $95^{\circ} \mathrm{C}$ for $30 \mathrm{~s}$, annealing at $60^{\circ} \mathrm{C}$ for $1 \mathrm{~min}$, extension at $72^{\circ} \mathrm{C}$ for $1 \mathrm{~min}$, and then final extension at $72^{\circ}$ for $1 \mathrm{~min}$ [13].

\section{Electrophoresis}

The PCR amplification products were analyzed using 1.5\% agarose gel stained with gel red. Electrophoresis was performed for $30 \mathrm{~min}$ at 400 $\mathrm{A}$ and $100 \mathrm{~V}$. Then, agarose gel was exposed to UV light.

\section{RESULTS}

The clinical parameters used in this study were oral hygiene status and organoleptic score. The correlation between $\mathrm{OHI}-\mathrm{S}$ and organoleptic score in nose breather group was a weak negative correlation $(\mathrm{r}=-0.046)$, meanwhile, in mouth breather group was a weak positive correlation $(r=0.001) . p>0.05$ is no significant correlation between $\mathrm{OHI}-\mathrm{S}$ and organoleptic score.

Overall, as shown in Table $1, T$. denticola and P. gingivalis were detected in every subject from the supragingival plaque and buccal mucosa. T. denticola was detected in $100 \%(\mathrm{n}=41)$ nose breathers when isolated from supragingival plaque and was detected $100 \%$ $(\mathrm{n}=19)$ in mouth breathers when isolated from buccal mucosa. Then, in buccal mucosa of mouth breathers, P. gingivalis was detected $100 \%$ $(\mathrm{n}=19)$. Meanwhile, T. denticola that isolated from supragingival plaque of mouth breathers and buccal mucosa of nose breathers was detected $89.5 \%$ and $92.7 \%$, respectively. The Chi-square test showed no statistically significant differences between nose and mouth breathers $(p>0.05)$.

The electrophoresis result from PCR amplification appears as a single band. According to the specific primers used in this study for each bacterium, T. denticola and P. gingivalis products yielded $105 \mathrm{bp}$ and $126 \mathrm{bp}$, respectively. Fig. 1 shows that all of subjects were detected T. denticola. Lane 13 does not contain T. denticola DNA because the DNA sample in PCR mixture was substitute with double-distilled water. Meanwhile, in Fig. 2, subject number 11 does not have P. gingivalis DNA because the band appears far lower than $126 \mathrm{bp}$.

The prevalence of $P$ gingivalis in both groups was same, but $T$. denticola has $90 \%$ tendency in halitosis group. Meanwhile, T. denticola prevalence in supragingival plaque's nose breathers showed a similar result in both of group halitosis (Fig. 3). While P. gingivalis has been increased in both group from supragingival plaque in nose breathers (Fig. 4). On the other hand, T. denticola and P. gingivalis have been

Table 1: T. denticola and $P$. gingivalis distribution in nose and mouth breathers

\begin{tabular}{|c|c|c|c|c|c|}
\hline \multirow[t]{2}{*}{ Bacteria } & \multirow[t]{2}{*}{ Sample } & \multicolumn{2}{|c|}{$\begin{array}{l}\text { Nose } \\
\text { breathers }\end{array}$} & \multicolumn{2}{|c|}{$\begin{array}{l}\text { Mouth } \\
\text { breathers }\end{array}$} \\
\hline & & $\mathbf{n}$ & $\%$ & n & $\%$ \\
\hline \multirow[t]{2}{*}{ T. denticola } & Supragingival plaque & 41 & 100 & 17 & 89.5 \\
\hline & Buccal mucosa & 38 & 92.7 & 19 & 100 \\
\hline \multirow{2}{*}{ P. gingivalis } & Supragingival plaque & 38 & 92.7 & 19 & 100 \\
\hline & Buccal mucosa & 39 & 95.1 & 18 & 94.7 \\
\hline
\end{tabular}

T. denticola: Treponema denticola, P. gingivalis: Porphyromonas gingivalis

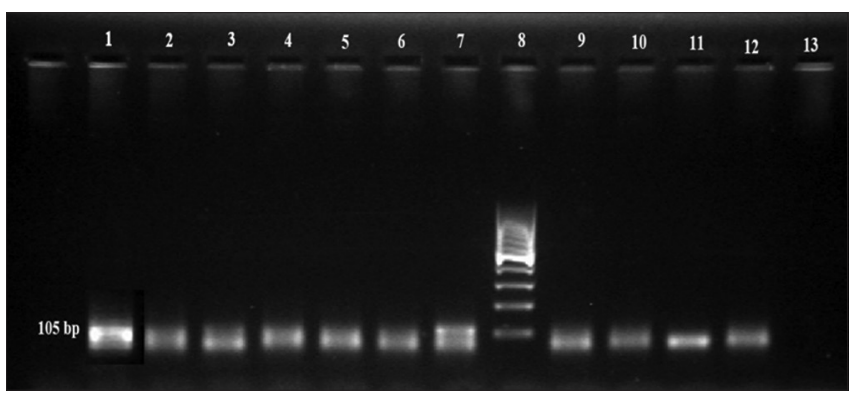

Fig. 1: Electrophoresis results from Treponema denticola amplification using a specific primer. Lane 1 DNA from T. denticola ATCC 35405. Lanes 2-7 and 9-12 DNA T. denticola from supragingival plaque subjects. Lane 13 does not contain T.denticola DNA. Marker is in lane 8

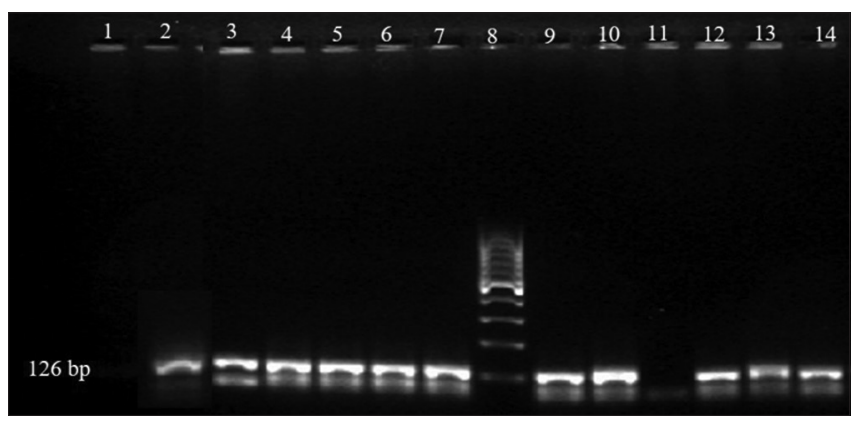

Fig. 2: Electrophoresis results from polymerase chain reaction amplification using a specific primer. Lane 1 was a control negative, which was not contained DNA sample. Lane 2 DNA from Porphyromonas gingivalis ATCC 33277. Lanes 3-7, 9, 10, and 12-14 DNA $P$. gingivalis isolated from supragingival plaque subjects. Lane 11 does not contain P. gingivalis DNA 


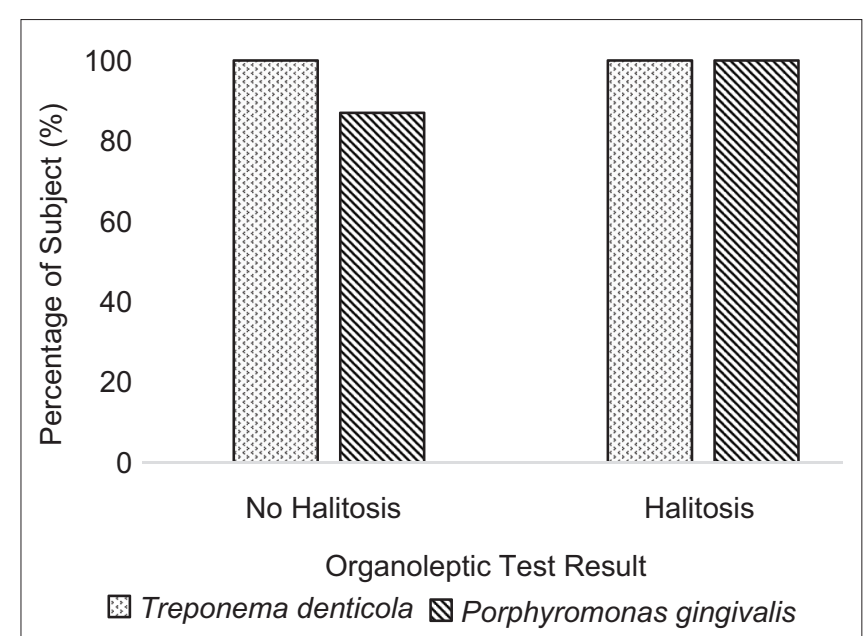

Fig. 3: Comparison identification positive DNA Treponema denticola and Porphyromonas gingivalis in supragingival plaque nose breathers based on organoleptic score

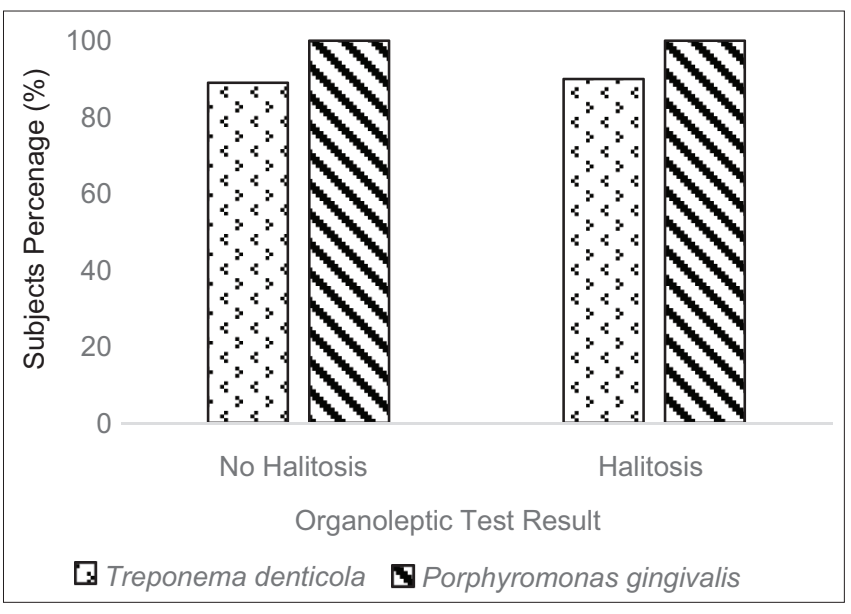

Fig. 4: Comparison identification positive DNA Treponema denticola and Porphyromonas gingivalis in supragingival plaque mouth breathers based on organoleptic score

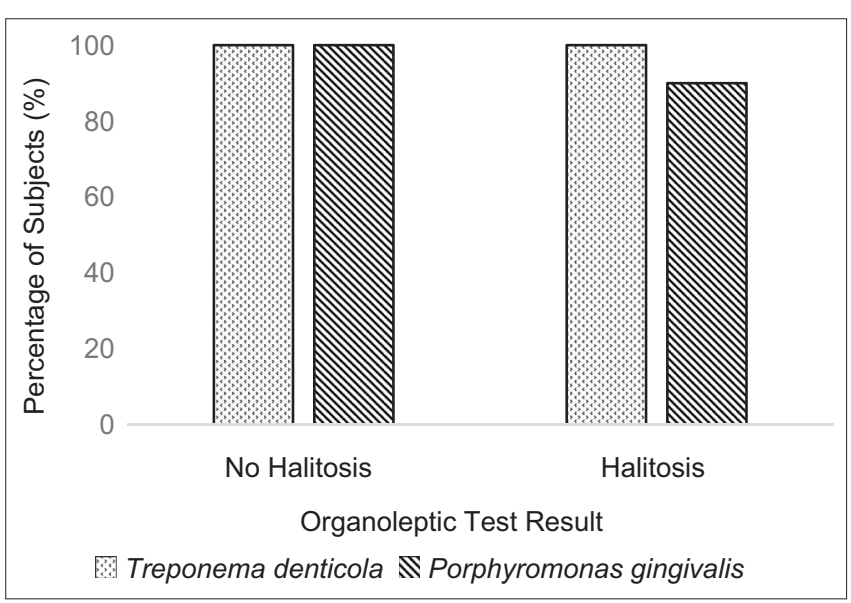

Fig. 5: Comparison identification positive DNA Treponema denticola and Porphyromonas gingivalis in buccal mucosa mouth breathers based on organoleptic score

found in the same level in the supragingival plaque in mouth breathers (Fig. 5). Meanwhile, both of bacteria that isolated from buccal mucosa, T. denticola and P. gingivalis, have higher prevalence in nose breather subject without halitosis (Fig. 6). The differences of T. denticola and P. gingivalis between halitosis and no halitosis groups were $7 \%$ and $2 \%$, respectively. Statistical analysis using Chi-square test was carried out for every group with different samples. $p>0.05$ is no statistically significant differences between the groups.

Furthermore, the mouth and nose breathers were classified by their oral hygiene status into good, moderate, and poor oral hygiene category. However, in this study, the poor oral hygiene category was not found among the subjects. P. gingivalis was detected in all of mouth breathers between good and moderate oral hygiene status. However, T. denticola showed a higher prevalence in mouth breather with moderate oral hygiene (Fig. 7). The differences between moderate and good oral hygiene are $6 \%$. In contrast, $T$. denticola isolated from supragingival plaque was detected in all of nose breather whether good or moderate oral hygiene status. However, $P$. gingivalis appear higher in moderate oral hygiene group (Fig. 8). The prevalence of T. denticola and $P$. gingivalis isolated from buccal mucosa mouth breather has shown a special phenomenon (Fig. 9). T. denticola and P. gingivalis were detected in all of mouth breather with moderate and poor oral hygiene. Last, in Fig. 10, the prevalence of $T$. denticola and P. gingivalis has similarity in each oral hygiene category.

Statistical analysis was carried out for each clinical parameter in every group. The result showed that there are no statistically differences between the groups ( $p>0.05)$.

\section{DISCUSSION}

The aim of this study was to determine whether the prevalence of $T$. denticola and $P$. gingivalis could be a biologic indicator in mouth breathing children or not. Mouth breathing is a pathological adaptation caused oral cavity alteration, which is halitosis and poor oral hygiene [3]. Those conditions occur due to decreased salivary flow rate leading to lack of its protection properties. Salivary antibacterial action could suppress the growth and proliferation of anaerobic Gram-negative bacteria [4].

Extraoral halitosis can originate from respiration and gastrointestinal system. Gastrointestinal system disorder can cause halitosis, including gastroesophageal reflux. According to Moskowitz et al., there was a relationship between gastrointestinal disease and halitosis. Furthermore, Katsinoles et al. had studied organoleptic and benzoylDL-arginine-test- $\alpha$-naphthylamide (BANA) test to evaluate halitosis in subjects positive and negative Helicobacter pylori. BANA test used to detect anaerobic bacteria proteolytic, especially $T$. denticola, P. gingivalis, and T. forsythia [6].

T. denticola and P. gingivalis were chosen to be detected because both bacteria are Gram-negative anaerobic proteolytic bacteria. Proteolytic bacteria will degrade protein from saliva, shed epithelium, food debris, and interdental plaque. Protein compounds were broken into peptide and then amino acid and VSC gas with thiol free group. As mentioned before, VSC gases contribute to halitosis occurrence [7].

In this study, 10 of 19 mouth breathers had halitosis. Mouth breathing causes oral dryness in oral mucosa due to inability saliva to balancing the evaporation. Thus, the oral cavity environment favored to the occurrence of halitosis. However, there are some nose breathers who had halitosis. It is assumed that halitosis does not originate from oral cavity rather extraoral [4]. This situation was supported by biologic indicators, almost all of the subjects were detected T. denticola and $P$. gingivalis. It is assumed that the accumulation of each bacterium was not quite dominant resulted in a lower concentration of VSC gas produced. This was supported by clinical parameter that no subjects were included poor oral hygiene category because halitosis related to poor oral hygiene and periodontal disease existence [8].

The prevalence of $T$. denticola and $P$. gingivalis whether from supragingival plaque or buccal mucosa was not showed significant differences (Table 1). Most of T. denticola are found in conjunction 


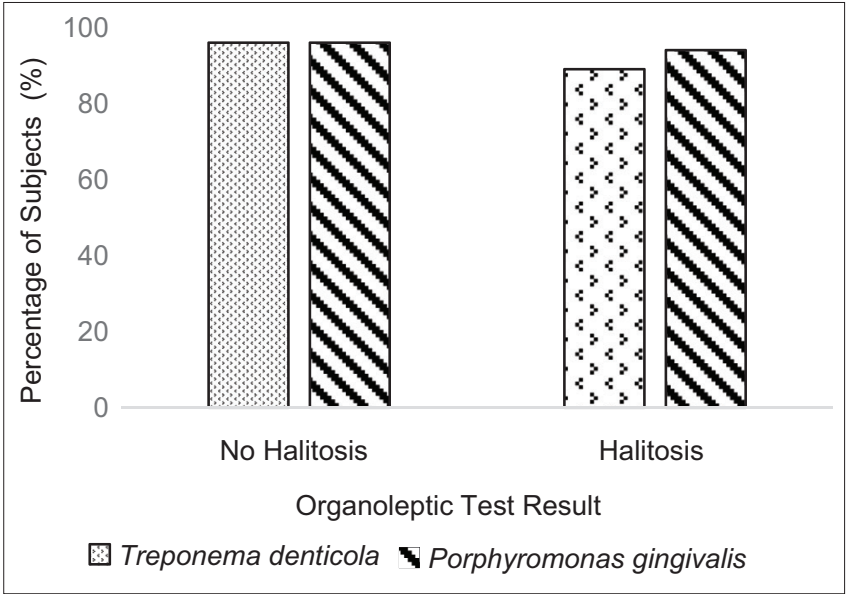

Fig. 6: Comparison identification positive DNA Treponema denticola and Porphyromonas gingivalis in buccal mucosa nose breathers based on organoleptic score

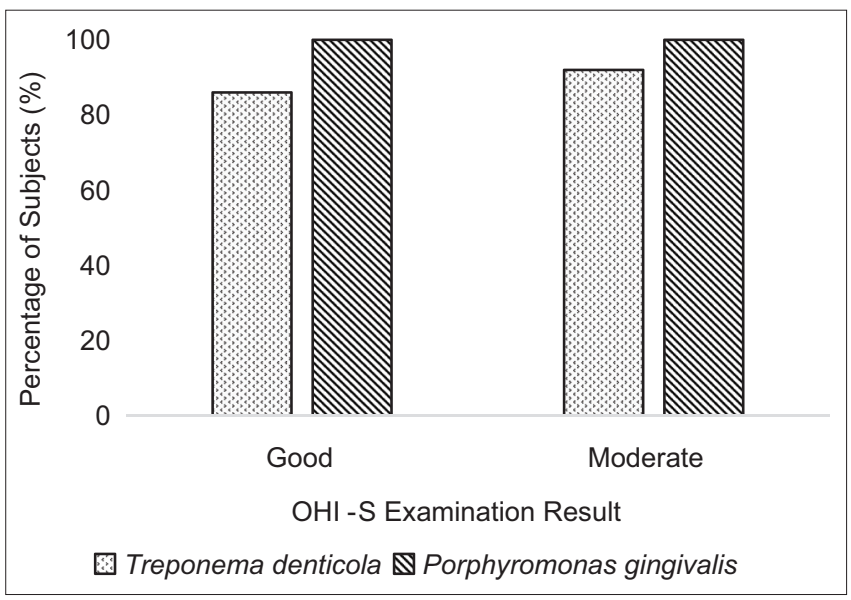

Fig. 7: Comparison identification positive DNA Treponema denticola and Porphyromonas gingivalis in supragingival plaque mouth breathers based on Oral Hygiene Index-Simplified category

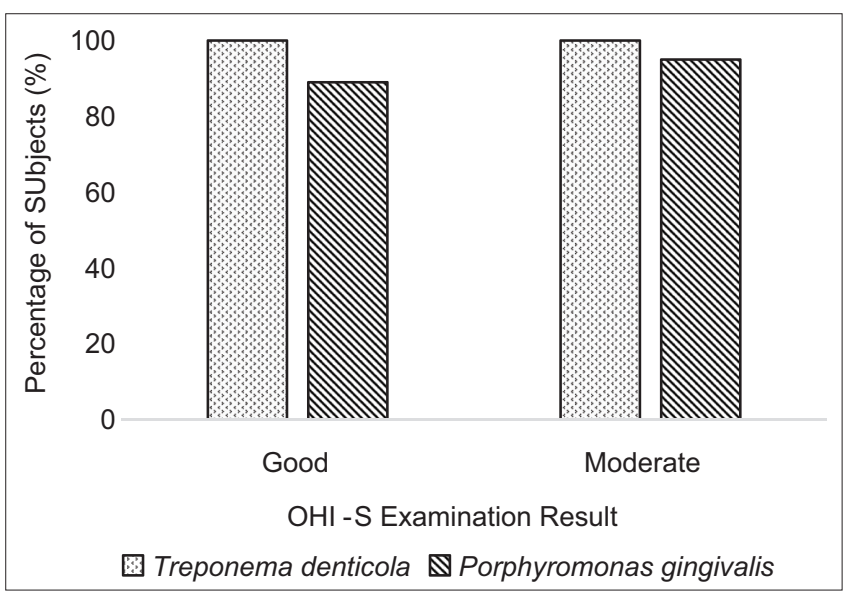

Fig. 8: Comparison identification positive DNA Treponema denticola and Porphyromonas gingivalis in supragingival plaque nose breathers based on Oral Hygiene Index-Simplified category

with P. gingivalis in supragingival plaque (data not shown). When the plaque formation occurred, both of bacteria act as late colonizer and they will coaggregate. Interaction between $T$. denticola and $P$. gingivalis is nutritional synergic symbiosis [14]. T. denticola will stimulate

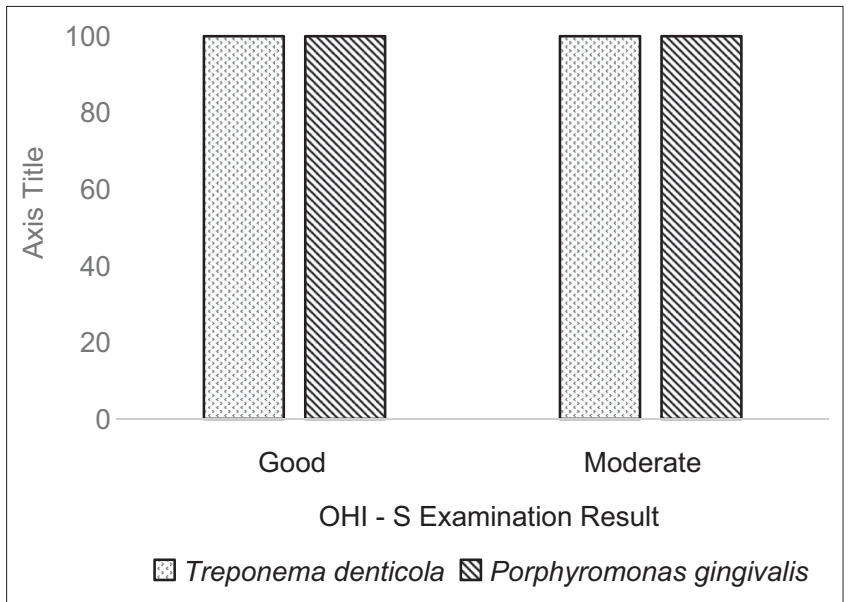

Fig. 9: Comparison identification positive DNA Treponema denticola and Porphyromonas gingivalis in buccal mucosa mouth breathers based on Oral Hygiene Index-Simplified category

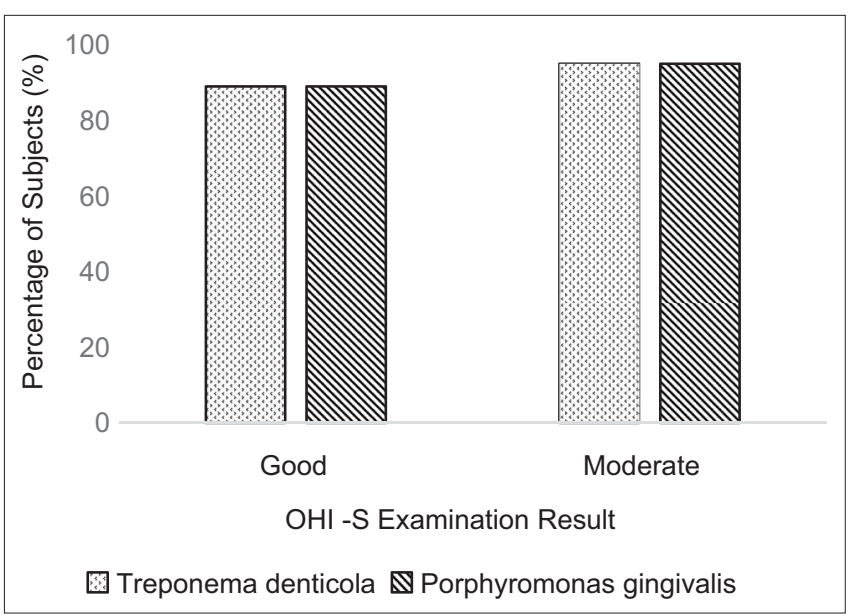

Fig. 10: Comparison identification positive DNA Treponema denticola and Porphyromonas gingivalis in buccal mucosa nose breathers based on Oral Hygiene Index-Simplified category

P. gingivalis to generate a free glycine. T. denticola was used free glycine by P. gingivalis as a main source of carbon [15]. Meanwhile, T. denticola has been reported that always found in periodontal tissue that infected by $P$. gingivalis [14].

However, subject number 2 (data not shown) had a contrary result. $P$. gingivalis was not found when isolated from buccal mucosa, but T. denticola was detected in the same area. T. denticola existence in buccal mucosa can cause by tongue movement or saliva. The more sample sources were collected it increased the possibility of bacteria to be found [16].

Bacterial existence in buccal mucosa was supported by the previous study that $P$. gingivalis was found in predentate infant [17]. It is proposed that $P$. gingivalis was transmitted through an intimate contact, including mother and child contact with direct transmission from the mother $[17,18]$. $P$. gingivalis mechanism to survive in an aerobic environment like buccal mucosa was not clear. However, it is believed that $P$. gingivalis invade into buccal mucosa cell. Under that circumstance, bacteria were protected against extracellular oxygen derived from saliva, saliva agglutinin, and antibacterial protein saliva [19].

According to Table 1, the prevalence of $P$. gingivalis from supragingival plaque in mouth breather is $100 \%$. Meanwhile, in nose breathers, subject number 13 and 29 (data not shown), P. gingivalis was detected in 
supragingival plaque but not detected in buccal mucosa. It is assumed, the supragingival plaque bacteria do not have a chance to colonize on buccal mucosa surface [20].

This study reported the prevalence of $T$. denticola and P. gingivalis from supragingival plaque and buccal mucosa each group had unstable tendency. The differences between breather groups with or without halitosis are also not significant. It is assumed that $T$. denticola and $P$. gingivalis do not correlate with halitosis. Perhaps, halitosis in these subjects originates from extraoral rather intraoral. One of the halitosis sources is gastroesophageal reflux. Alteration in gastric caused by H. pylori infection. Katsinoles et al. study was carried out an examination of halitosis using BANA test in positive and negative subjects $H$. pylori. The result showed a higher BANA positive in children positive $H$. pylori in their oral cavity. Another study by Hoshi et al. showed higher $\mathrm{H}_{2} \mathrm{~S}$ and $\left[\left(\mathrm{CH}_{3}\right)_{2} \mathrm{SH}\right]$ concentration in subjects positive $H$. pylori. Therefore, H. pylori had a greater relationship to halitosis in children [4].

The evaluation of oral hygiene status was carried out in this study. According to Figs. 7-10, T. denticola and P. gingivalis have tendencies in moderate OHI-S group. Tanaka et al. study supported this result, the prevalence of both bacteria was found higher in moderate OHI-S group [18]. There are no significant differences between the OHI-S groups. T. denticola and $P$. gingivalis were found almost in every subject with different oral hygiene status. This might be happened because $T$. denticola and $P$. gingivalis act as late colonizer in plaque formation [10]. Besides, this is a qualitative study, so T. denticola and $P$. gingivalis identification will be needed a further study by calculate the amount of colonization both bacteria in every oral hygiene status.

Biological indicator that analyzed in this study, T. denticola and $P$. gingivalis, did not correlate with halitosis. The halitosis sources might be originated from gastroesophageal reflux. For further study, the identification of $H$. pylori could carry out as bacteria that more correlate with halitosis. This study has several limitations, including the distribution of sample sizes between nose and mouth breathers was not equal. Besides, this is a qualitative study so that the quantity of bacterial colonization could not be carried out. Therefore, real-time PCR method is needed to examine the quantity of bacterial colonization.

\section{CONCLUSION}

The prevalence of $T$. denticola and $P$. gingivalis based on oral hygiene status and organoleptic score from supragingival plaque and buccal mucosa in mouth and nose breathers has no constant tendencies. Therefore, T. denticola and P. gingivalis isolated from supragingival plaque and buccal mucosa cannot be used as biologic indicator in mouth breathing children based on oral hygiene status and organoleptic score.

\section{ACKNOWLEDGMENT}

The authors are grateful to Professor Boy Muchlis Bachtiar for the kind help and support throughout this study. The publication of this manuscript is supported by Universitas Indonesia.

\section{CONFLICTS OF INTEREST}

There are no conflicts of interest.

\section{REFERENCES}

1. Morais-Almeida M, Wandalsen GF, Solé D. Growth and mouth breathers. J Pediatr (Rio J) 2019;95 Suppl 1:66-71.

2. Harari D, Redlich M, Miri S, Hamud T, Gross M. The effect of mouth breathing versus nasal breathing on dentofacial and craniofacial development in orthodontic patients. Laryngoscope 2010;120:2089-93.

3. Triana CB, Ali AH, León IB. Mouth breathing and its relationship to some oral and medical conditions: Physiopathological mechanisms involved. Rev Habanera Cien Med 2016;15:200-12.

4. Motta LJ, Bachiega JC, Guedes CC, Laranja LT, Bussadori SK. Association between halitosis and mouth breathing in children. Clinics (Sao Paulo) 2011;66:939-42.

5. Villa A, Zollanvari A, Alterovitz G, Cagetti MG, Strohmenger L, Abati S. Prevalence of halitosis in children considering oral hygiene, gender and age. Int J Dent Hyg 2014;12:208-12.

6. Bicak DA. A current approach to halitosis and oral malodor a mini review. Open Dent J 2018;12:322-30.

7. Saini N. Oral malodor: A common oral problem. J Bioeng Biomed Sci 2012;2:1-7.

8. De Geest S, Laleman I, Teughels W, Dekeyser C, Quirynen M. Periodontal diseases as a source of halitosis: A review of the evidence and treatment approaches for dentists and dental hygienists. Periodontol 2000 2016;71:213-27.

9. Reinhardt B, Klocke A, Neering SH, Selbach S, Peters U, Flemmig TF, et al. Microbiological dynamics of red complex bacteria following fullmouth air polishing in periodontally healthy subjects a randomized clinical pilot study. Clin Oral Investig 2019;23:3905-14.

10. Valen H, Biofilms SA. Biofilms and their properties. Eur J Oral Sci 2018;126:13-8.

11. Greene JC, Vermillion JR. The simplified oral hygiene index. J Am Dent Assoc 1964;68:7-13.

12. Kato H, Yoshida A, Awano S, Ansai T, Takehara T. Quantitative detection of volatile sulfur compound-producing microorganisms in oral specimens using real-time PCR. Oral Dis 2005;11 Suppl 1:67-71.

13. Yoshida A, Suzuki N, Nakano Y, Oho T, Kawada M, Koga T. Development of a 5' fluorogenic nuclease-based real-time PCR assay for quantitative detection of Actinobacillus actinomycetemcomitans and Porphyromonas gingivalis. J Clin Microbiol 2003;41:863-6.

14. Kolenbrander PE, London J. Minireview adhere today, here tomorrow: Oral bacterial adherence. J Bacteriol 1993;175:3247-52.

15. Tan KH, Seers CA, Dashper SG, Mitchell HL, Pyke JS, Meuric V, et al. Porphyromonas gingivalis and Treponema denticola exhibit metabolic symbioses. PLoS Pathog 2014;10:e1003955.

16. Lamell CW, Griffen AL, McClellan DL, Leys EJ. Acquisition and colonization stability of Actinobacillus actinomycetemcomitans and Porphyromonas gingivalis in children. J Clin Microbiol 2000;38:1196-9.

17. McClellan DL, Griffen AL, Leys EJ. Age and prevalence of Porphyromonas gingivalis in children. J Clin Microbiol 1996;34:2017-9.

18. Tanaka S, Murakami Y, Seto K, Takamori K, Yosida M, Ochiai K, et al . The detection of Porphyromonas gingivalis, Prevotella intermedia, and Actinobacillus actinomycetemcomitans in the supragingival plaque of children with and without caries. Pediatr Dent 2003;25:143-8.

19. Pacheco MC, Casagrande CF, Teixeira LP, Finck NS, de Araújo MT. Guidelines proposal for clinical recognition of mouth breathing children. Dental Press J Orthod 2015;20:39-44.

20. Wei Y, Shi M, Zhen M, Wang C, Hu W, Nie Y, et al. Comparison of subgingival and buccal mucosa microbiome in chronic and aggressive periodontitis: A pilot study. Front Cell Infect Microbiol 2019;9:53 